\title{
Prefrontal AMPA receptors are involved in the effect of methylphenidate on response inhibition in rats
}

\author{
Dong-dong ZHANG, Yu-qiu ZHANG, Xue-han ZHANG* \\ Institutes of Brain Science and State Key Laboratory of Medical Neurobiology, Fudan University, Shanghai 200032, China
}

\begin{abstract}
Response inhibition is a critical executive control function in many species. Deficits in response inhibition have been observed in many disorders, eg, attention deficit/hyperactivity disorder (ADHD). The stop-signal task (SST) is a unique behavior task for evaluating response inhibition via measuring the covert latency of a stop process, and it is widely used in studies of humans, nonhuman primates and rodents. Methylphenidate (MPH; Ritalin $\left.{ }^{\circledR}\right)$ is a psychostimulant that is widely used for the treatment of ADHD and that effectively improves response inhibition in individuals with ADHD and normal subjects. However, its mechanism of improving response inhibition remains unknown. In this study we adopted a rodent nose-poking version of the SST to examine response inhibition by estimating the stop signal reaction time (SSRT) in rats. Administration of MPH (1 mg/kg, sc) $25 \mathrm{~min}$ before the SST test exerted a baseline-dependent effect of MPH on response inhibition, ie, it shortened the SSRTs only in the rats with larger baseline SSRTs, thereby improving response inhibition in these rats. The effect of MPH on response inhibition remained $3 \mathrm{~h}$ after MPH administration. Co-administration of PP2 (1 $\mathrm{mg} / \mathrm{kg}, \mathrm{sc}$ ), a Src-protein tyrosine kinase (Src-PTKs) inhibitor that inhibited the upregulation of glutamate receptor expression on the plasma membrane of the prefrontal cortex (PFC), abolished the MPH-caused improvement in response inhibition. Furthermore, intraPFC infusion of a selective AMPAR antagonist. NASPM $(0.3 \mathrm{mmol} / \mathrm{L}$, per side $)$ via stainless guide cannulas implanted earlier abolished the effect of MPH on SSRT. These results suggest that AMPA receptors in the PFC are involved in the effect of MPH on response inhibition in rats.
\end{abstract}

Keywords: response inhibition; stop-signal task; prefrontal cortex; AMPA receptors; methylphenidate; SSRT; PP2; NASPM

Acta Pharmacologica Sinica (2018) 39: 607-615; doi: 10.1038/aps.2017.138; published online 21 Dec 2017

\section{Introduction}

Response inhibition, an important adaptive capability, instructs subjects to stop inappropriate behaviors under a sudden change of environment ${ }^{[1]}$. Deficits in response inhibition have been reported in many disorders, such as drug abuse, obsessive-compulsive disorder, schizophrenia, and especially attention deficit/hyperactivity disorder (ADHD) ${ }^{[2-5]}$.

Methylphenidate (MPH; Ritalin ${ }^{\circledR}$ ) is a psychostimulant widely used for the treatment of $\mathrm{ADHD}^{[6,7]}$. A therapeutic dose of $\mathrm{MPH}$ improves inhibitory control in individuals with $\mathrm{ADHD}^{[8-11]}$ as well as in normal human subjects and animals ${ }^{[9,10,12,13]}$. As a transporter inhibitor, MPH blocks the dopamine transporter (DAT) and norepinephrine transporter (NET) and preferentially elevates the concentrations of dopamine and norepinephrine at synapses in the prefrontal cortex (PFC) ${ }^{[14-16]}$; however, the mechanisms by which a therapeutic dose of $\mathrm{MPH}$ acutely improves response inhibition are unclear.

\footnotetext{
${ }^{*}$ To whom correspondence should be addressed. E-mail xuehanzhang@fudan.edu.cn

Received 2017-05-22 Accepted 2017-08-01
}

The PFC is a key brain region mediating cognitive and executive functions, including working memory, sustained attention, cognitive flexibility, and inhibitory control ${ }^{[17-20]}$. Malfunctions in the PFC have been related to deteriorated response inhibition ${ }^{[21-25]}$. Imaging studies have shown that $\mathrm{MPH}$ can change the neuronal activity of the cortex during the stop-signal task ${ }^{[26-28]}$. The effect of $\mathrm{MPH}$ on cognitive function is dependent on glutamate receptors in the $\mathrm{PFC}^{[29]}$. Response inhibition requires coordinated activities between the PFC and subcortical brain regions, such as the striatum, global pallidus and subthalamic nucleus ${ }^{[30-33]}$. Top-down control of the PFC depends on the output neurons within it. Glutamatergic neurotransmissions participate in many brain functions ${ }^{[34]}$ and are key to the output neurons in the PFC; however, it is unknown whether glutamate receptors in the PFC play a role in the effect of $\mathrm{MPH}$ on response inhibition.

In the present study, we used a rodent nose-poking stop-signal task (SST) to estimate the stop signal reaction time (SSRT), which reflects the capacity of response inhibition control ${ }^{[21,35]}$. We examined the effect of $\mathrm{MPH}$ on response inhibition and the contribution of glutamate receptors in the rat PFC to this 
process.

\section{Materials and methods \\ Animals}

Male Sprague-Dawley rats (160-180 g) were purchased from SLACC (Shanghai, China). All rats were group-housed under a 12:12 light/dark cycle (light on at 8:00 am). Food and water were available ad libitum. The rats were weighed daily to ensure maintenance of $\sim 95 \%$ of their original body weights. All of the experimental procedures were conducted in accordance with the National Institutes of Health Guide for the Care and Use of Laboratory Animals (NIH Publications No. $80-23,1996)$ and were approved and monitored by the Ethical Committee of Animal Experiments at the Institutes of Brain Science, Fudan University (Shanghai, China).

\section{The stop-signal task Chamber}

The chamber had 3 nose-poke ports of $30 \mathrm{~mm}$ in diameter, which were positioned $50 \mathrm{~mm}$ above the chamber floor and were arranged horizontally $50 \mathrm{~mm}$ apart. The port in the middle served as a reward port, delivering a drop of water for each correct trial. Nose entries into any of the three ports were monitored with infrared detectors. The light above the center port (reward light) signaled reinforcement delivery, whereas the lights located above the other two ports signaled the availability of these ports for nose-poking. A separated tone generator was placed in the chamber. A microcontroller (Arduino) was used to control the training box, and the behavior data were acquired in customized software written in Python.

\section{Training procedure}

The stop-signal task (SST) for rats has been previously described ${ }^{[21]}$. On the first day, the rats were allowed to explore freely in the chamber for $1 \mathrm{~h}$ with all of the ports closed and the lights off. Then, the middle reward port was opened. When the reward light was on, a drop of water reward was delivered if the rats broke the infrared light beam in the reward port. The inter-trial interval (ITI) was $5 \mathrm{~s}$. When the rats were able to finish 100 trials in $30 \mathrm{~min}$, one of the two side ports (action port) opened, and the light above it (action light) was switched on. If the rats inserted their snouts into the action port, the action light turned off, and the reward light turned on. Collection of the water reward resulted in the action light coming back on. The action light remained on until the rats inserted their snouts into the action port to start a new trial. After the animals were able to complete a one-day session of 200 trials within $30 \mathrm{~min}$, the other side port (initial port) was introduced. The rats were trained to insert their snouts into the initial port to start a trial, then to insert their snouts into the action port (Go Response) within a limit hold $(\mathrm{LH})$ of $30 \mathrm{~s}$. If the rats failed to insert their snouts into the action port at the end of the $\mathrm{LH}$, they would receive a timeout punishment ( $5 \mathrm{~s}$ of darkness and no reward). The LH was progressively shortened. The time elapsed between leaving the initial port and nose-poking into the action port was defined as the go trial reaction time (Go RT). When the rats were able to perform two consecutive daily sessions of 300 trials in 30 min with an LH of $3 \mathrm{~s}$, the stop signal was introduced. The stop signal was an auditory stimulus ( $4000 \mathrm{~Hz}, \sim 60-\mathrm{dB}$ tone) that lasted $100 \mathrm{~ms}$ during the period of $\mathrm{LH}$ and was pseudorandomly presented in approximately $20 \%$ of the total trials. The stop trial was initiated in the same fashion as the go trial, but the stop signal was presented after the rats left the initial port. If the rats refrained from inserting their snouts into the action port during the LH, the water reward was delivered. Otherwise, they experienced a timeout. During the training, the stop signal was presented immediately at the start of the LH (Zero Delay, ZD). When stable performance of the task was achieved (the performances of the go trial and stop trial were over $90 \%$ in a 320 -trial session), behavioral testing was carried out.

\section{Testing procedure}

The testing session was composed of one adaptive block of 20 trials and three testing blocks of 100 trials each. During testing, the stop signal was delivered at variable delays (stop signal delay, SSD). We used a dynamic tracking procedure to achieve a $50 \%$ correction of the total stop trials. The initial SSD was set to be the median value of the Go RT in the adaptive block minus $200 \mathrm{~ms}$. In the following testing blocks, 20 stop trials were randomly interleaved in each block, and the SSD was constantly adjusted after every stop trial depending on the outcome: when the stop trial was successful, the SSD was increased by $50 \mathrm{~ms}$ for the next stop trial; otherwise, the SSD was decreased by $50 \mathrm{~ms}$. This tracking method resulted in approximately $50 \%$ accuracies for the stop trials.

\section{SSRT estimation}

The stop signal reaction time (SSRT) was estimated according to the independent-race model, whereby performance in the stop-signal task is modeled as a race between two independent internal processes ${ }^{[3]}$. The SSRT was calculated for each block separately and then averaged to yield a final estimate for each rat in one testing session. To ensure the accuracy of the SSRT estimation, we used QQ-plot to evaluate the normality of the Go RT (Figure 1C), excluding those with heavy tails.

\section{Surgery}

The rats were anesthetized with isoflurane and fixed in a stereotaxic apparatus (Stoelting, USA). Stainless guide cannulas (23-gauge) were implanted bilaterally into the location above the prelimbic (PL) area of the PFC (AP $3.3 \mathrm{~mm}$, ML 0.7 $\mathrm{mm}$, and DV $1.5 \mathrm{~mm}$ to the skull surface). Dummy cannulae were inserted into the guide cannula to prevent clotting and decrease the risk of infection. The rats were allowed a minimum recovery of $5 \mathrm{~d}$ before behavioral training.

\section{Drug administration}

Intra-PFC infusions were performed using 30-gauge infusion cannulas. The length of the infusion cannulas was $2 \mathrm{~mm}$ longer than the guide cannulas, thus yielding a total distance of 

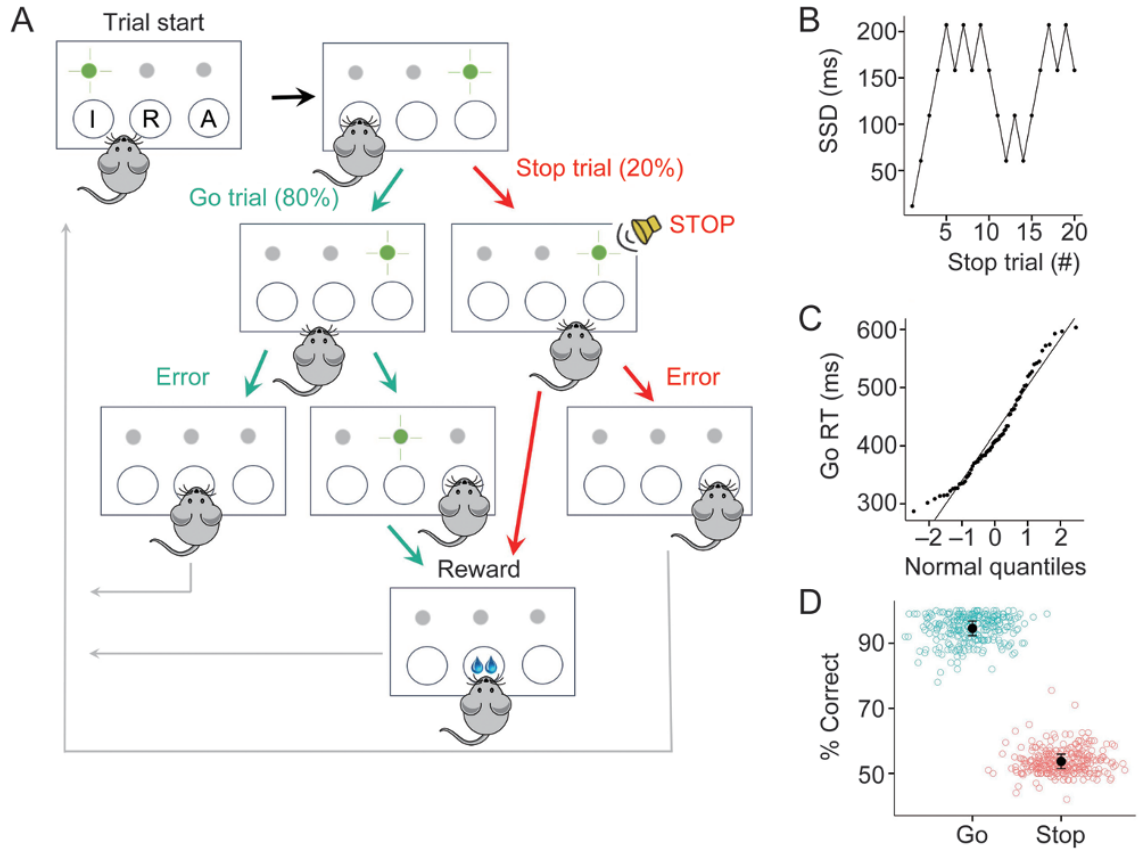

Figure 1. Stop-signal task setup and performance of rats in the study. (A) Schematic illustration of the testing procedure in the stop-signal task. In $80 \%$ of the trials (Go trials), rats nose-poked from port I to port A and received a water reward at port R. In a subset of $20 \%$ (Stop trials), a stop tone was presented at a variable delay after leaving port I, which indicated that the rats should suppress nose-poking into port A. (B) Change in stop signal delay (SSD) in one exemplary testing block. The SSD was changed dynamically according to the result of the previous stop trial and reached a plateau after several trials. (C) Go reaction time (Go RT) in the same exemplary testing block as shown in (B). The distribution of the Go RT was approximately normal. (D) Overall performance of go trials and stop trials for all the rats involved in the present study. Each point represents the performance of one rat in one session. The dots in the center are the means \pm SEMs.

$3.5 \mathrm{~mm}$ from the skull surface. All infusions were performed using a Hamilton syringe at a rate of $0.2 \mu \mathrm{L} / \mathrm{min}$. The total volume of drug delivered was $0.5 \mu \mathrm{L}$ per side. The infusion cannulas were left in place for an additional $2 \mathrm{~min}$ for drug diffusion.

\section{Histology}

After the behavior testing, the rats received trans-cardial perfusion with $4 \%$ paraformaldehyde (PFA). The brains were then removed and placed into 30\% sucrose for dehydration. Coronal sections $(30 \mu \mathrm{m})$ were cut using a cryostat (Leica). The guide cannulas and infusion sites were examined using a fluorescence microscope (Olympus, Japan).

\section{Data analysis}

The data are expressed as the mean \pm SEM, with $P<0.05$ indicating significance. Statistical analysis of the drug-induced difference was tested by the paired Wilcoxon rank sum test. When the SSRTs were normalized to range [0,1], the difference was statistically analyzed using the non-parametric permutation test.

\section{Results}

\section{Effect of MPH on response inhibition in rats}

We adopted a nose-poking version of the stop-signal task (SST) to evaluate the stop signal reaction time (SSRT) of the rats (Figure 1A). For $20 \%$ of the trials, a stop signal was deliv- ered between the rat leaving the initial port (I) and arriving at an action port (A), thus indicating that the rat should withhold the current action and not insert its snout into port A. Figure $1 \mathrm{~B}$ and $\mathrm{C}$ shows an example testing block from the same rat. The stop signal delays (SSDs) were changed dynamically and reached a plateau value after a few stop trials (Figure 1B), thereby achieving an overall 50\% stop accuracy (Figure 1D). The distribution of go reaction time (Go RT) was approximately normal during one testing block (Figure 1C), thus indicating that the rat responded instantly after the trial began and did not change strategies during the task (Figure 1C). The behavior accuracies for all the rats enrolled in the present study were approximately $90 \%$ and $50 \%$ for the go trial and stop trial, respectively (Figure 1D).

To assess the effect of MPH on response inhibition in rats, we first measured rat SSRTs one day before drug testing to obtain the baseline SSRT for each rat. The following day, the SSRTs were measured $25 \mathrm{~min}$ after MPH $(1.0 \mathrm{mg} / \mathrm{kg})$ was administered subcutaneously (Figure 2A). To evaluate MPHinduced changes in SSRTs, we calculated the differences in the SSRTs before and after MPH treatment. We found that the $\mathrm{MPH}$-induced change in the SSRTs was correlated with the baseline SSRTs (Figure 2B, $P<0.001$ ). As shown in Figure 2B, the rats with longer baseline SSRTs (eg, longer than the median value of all rat SSRTs) exhibited lower SSRTs after MPH infusion, whereas the rats with baselines shorter than the median had a tendency to exhibit increased SSRTs. Then, the rats were 
A

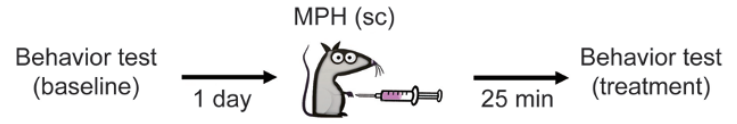

B

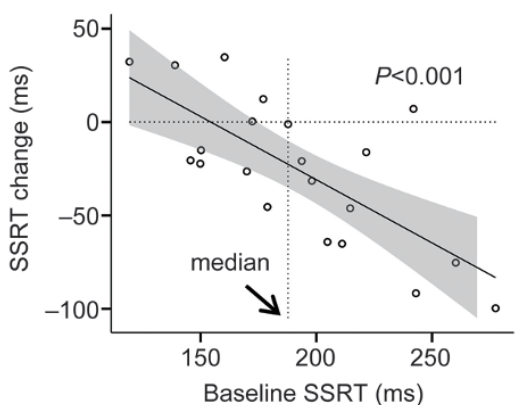

C

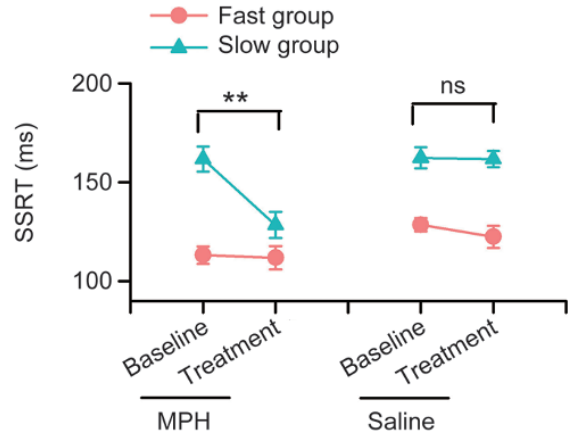

D

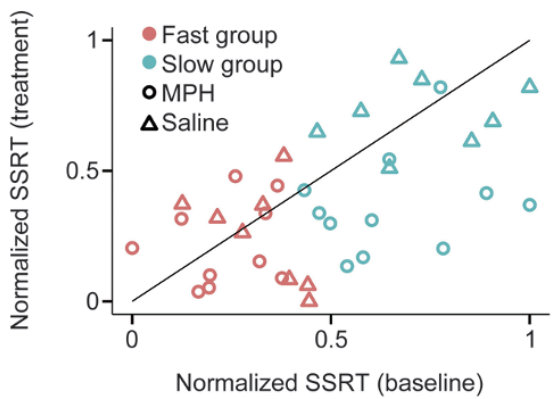

E

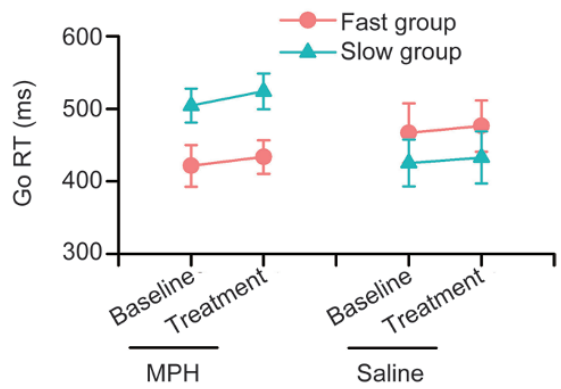

F

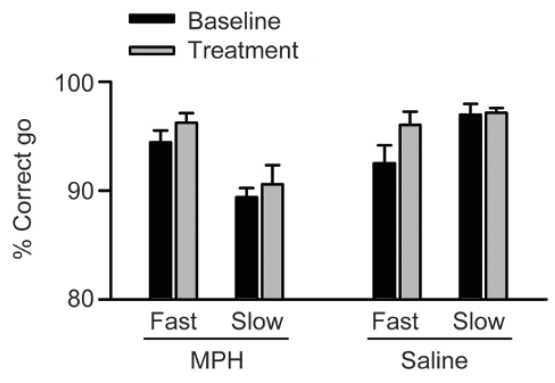

G

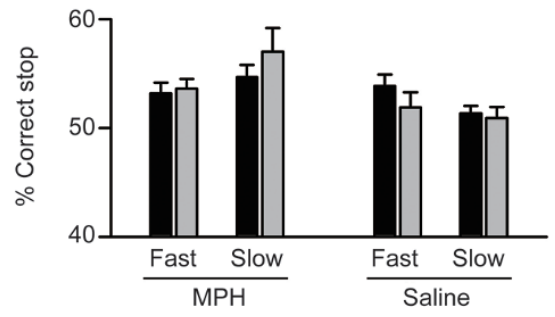

Figure 2. Effects of MPH on response inhibition in rats. (A) Scheme of the experimental procedure. (B) Linear regression of the stop signal reaction time (SSRT) before MPH infusion (baseline SSRT) with the SSRT change induced by MPH. The SSRT change was the difference in the SSRT before and after MPH infusion $(1.0 \mathrm{mg} / \mathrm{kg}$ ). The arrow indicates the median value of the baseline SSRTs. The shaded area represents the 95\% Cl. Each dot represents one rat $(n=21)$. $P<0.001$, linear regression. (C) Effects of MPH on response inhibition in rats with different baseline SSRTs. The Fast group (baseline SSRT<median) and the Slow group (baseline SSRT>median) were grouped according to the median of the baseline SSRTs. The MPH dataset is the same as that in (A). $n=10-11 .{ }^{* *} P<0.01$, paired Wilcoxon test. (D) Plot of normalized SSRT before (baseline) versus after MPH or saline infusion (treatment) for each rat. $P<0.01$ for the Slow group with MPH treatment (green circles), permutation test. (E-G) Go reaction time (Go RT) (E), go accuracy $(F)$, and stop accuracy $(G)$ were kept intact in both the Fast group and the Slow group after MPH or saline infusion.

divided into two groups for subsequent analysis. We defined rats with longer baseline SSRTs (>median) as the Slow group and rats with shorter baseline SSRTs (<median) as the Fast group. Figure 2C shows that MPH significantly shortened the SSRTs of the Slow group $(P<0.01, n=11)$ and did not affect the SSRTs of the Fast group $(P=0.67, n=10)$. The SSRTs of both the Slow group $(n=8)$ and the Fast group $(n=8)$ were unchanged after saline infusion (Figure 2C). The MPH-induced changes in the SSRTs were also demonstrated using normalized SSRTs before versus after MPH treatment. As shown in Figure 3D, the MPH-treated, Slow group was mainly distributed below the diagonal $(P<0.01)$, whereas others were evenly distributed along the diagonal $(P>0.05)$. No changes in the Go RT (Figure $2 \mathrm{E}$ ), go accuracy (Figure 2F), or stop accuracy (Figure 2G) were observed after $\mathrm{MPH}$ versus saline administration in either group $(P>0.05)$, thus indicating that $\mathrm{MPH}$ had no effect on the action response and behavior strategy of the rats during the task.

These results demonstrated that MPH exerted a baselinedependent effect on response inhibition in rats and improved the response inhibition of only rats with greater SSRTs (Slow 
A

A 25 min post-MPH

25 min post-MPH $3 \mathrm{~h}$ post-MPH

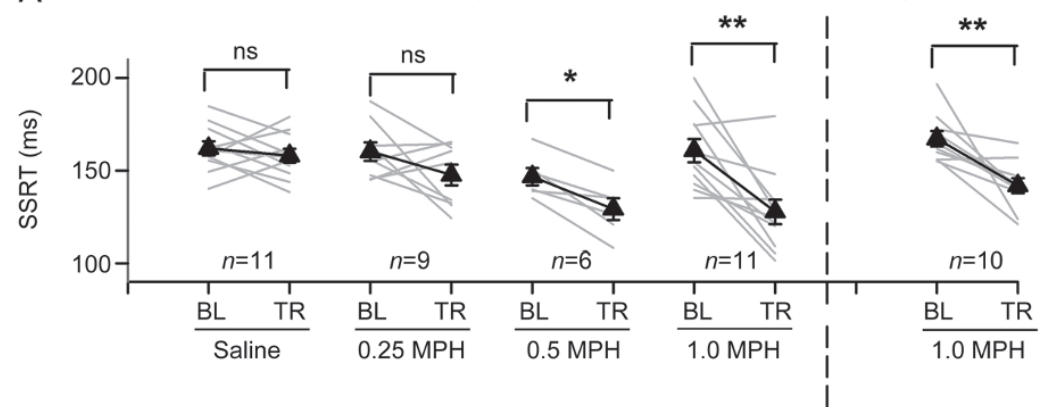

B

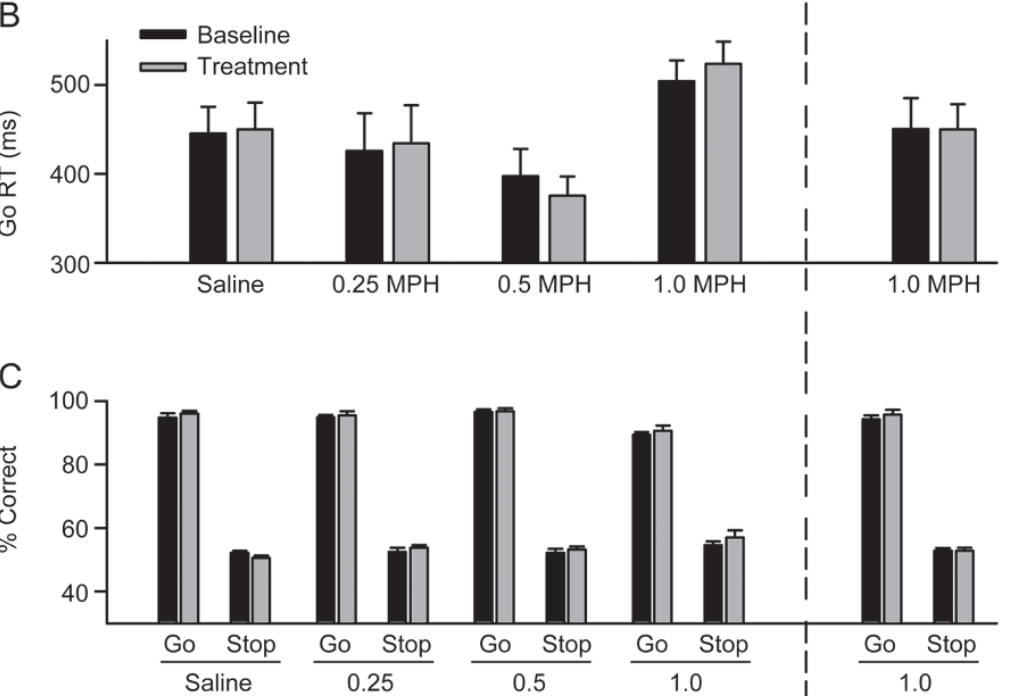

Figure 3. Effects of different doses of MPH on response inhibition. Effects of MPH at $0.25,0.5$, and $1.0 \mathrm{mg} / \mathrm{kg}$ on the SSRTs of rats with longer SSRTs (Slow group) at $25 \mathrm{~min}$ or $3 \mathrm{~h}$ after MPH infusion. The data for $25 \mathrm{~min}$ after infusion with $1 \mathrm{mg} / \mathrm{kg} \mathrm{MPH}$ are presented here. Each gray line represents one rat. " $P<0.05,{ }^{* *} P<0.01$, paired Wilcoxon test. $B L$, baseline; TR, treatment. (B and $\mathrm{C}$ ) Go reaction time (Go RT) (B) and trial accuracies $(\mathrm{C})$ were not altered after MPH infusion.

group), in agreement with findings from a previous report ${ }^{[13]}$. Thus, we focused on the Slow SSRT group in the subsequent analyses.

\section{Dose-effects of MPH on response inhibition}

To test the effects of different doses of MPH on response inhibition, we assessed the SSRTs of rats with longer SSRTs (Slow group) at $25 \mathrm{~min}$ after MPH $(0.25,0.5$, or $1.0 \mathrm{mg} / \mathrm{kg})$ infusion. As shown on the left of Figure 3, the SSRTs of the Slow group were significantly decreased by $0.5 \mathrm{mg} / \mathrm{kg}(P<0.05, n=6)$ and $1.0 \mathrm{mg} / \mathrm{kg} \mathrm{MPH}(P<0.01, n=11)$ but not $0.25 \mathrm{mg} / \mathrm{kg} \mathrm{MPH}$ $(P>0.05, n=9)$. When behavioral testing was extended to $3 \mathrm{~h}$ after MPH infusion $(1.0 \mathrm{mg} / \mathrm{kg})$, the SSRTs of the Slow group were still dramatically decreased $(P<0.01, n=10$; Figure $3 \mathrm{~A}$, right), thus suggesting that the effect of $\mathrm{MPH}$ lasted more than $3 \mathrm{~h}$. Similarly, the Go RT (Figure 3B) and behavior accuracy (Figure 3C) of the Slow group were unaffected by these conditions $(P>0.05)$.

PP2 abolishes the MPH-induced improvement in response inhibition

$\mathrm{MPH}$ has been proposed to potentiate glutamate receptor expression and function, thereby enhancing PFC-mediated cognition functions ${ }^{[29]}$. To determine whether the effect of $\mathrm{MPH}$ on response inhibition depends on this mechanism, we used PP2 to block the MPH-induced potentiation of glutamate receptors and then examined the effect of $\mathrm{MPH}$ on the SSRTs of rats with longer SSRTs (Slow group) (Figure 4A). PP2 is a Src-protein tyrosine kinase (Src-PTKs) inhibitor that can occlude glutamate receptor transport from the cytoplasm to the cell surface or accelerate glutamate receptor endocytosis by inhibiting tyrosine residue phosphorylation ${ }^{[36,37]}$. As shown in Figure 4, PP2 $(1.0 \mathrm{mg} / \mathrm{kg})$ alone had no effect on the SSRTs of the Slow group $(P>0.05, n=8$; Figure $4 \mathrm{~B})$; however, when $\mathrm{MPH}(1.0 \mathrm{mg} / \mathrm{kg})$ was co-infused with PP2 $(1.0 \mathrm{mg} / \mathrm{kg})$, the effect of MPH on the SSRTs of the Slow group was abolished $(P>0.05, n=6$; Figure 4B). Normalized SSRTs measured before $\mathrm{MPH}$ infusion compared with those measured after MPH infusion in rats pre-treated with PP2 showed no significant change $(P>0.05$; Figure $4 \mathrm{C})$. Similarly, the Go RT (Figure 4D) and behavior accuracy (Figure 4E) of the Slow group were not changed by these treatments $(P>0.05)$.

These results suggested that the effect of MPH on response inhibition may also involve the potentiation of glutamate 


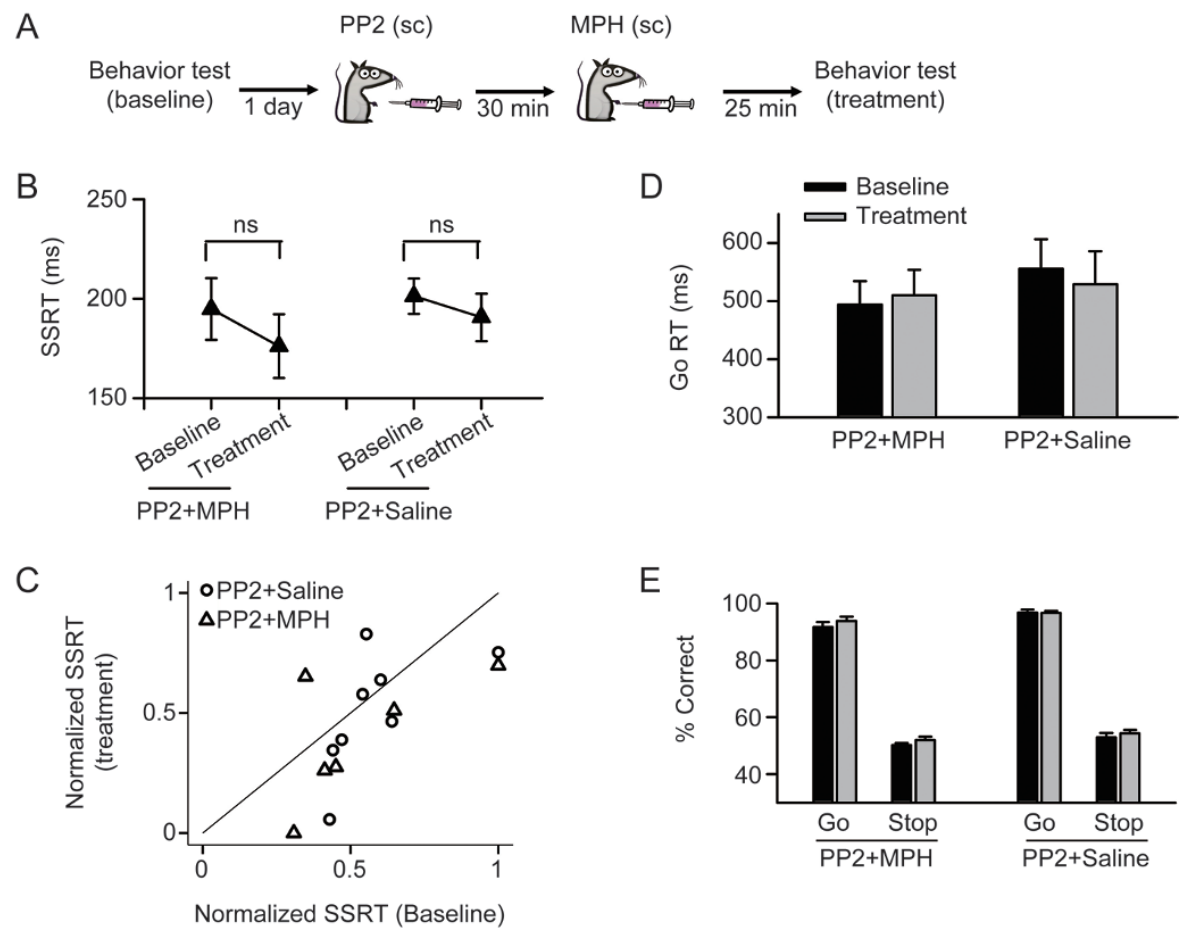

Figure 4. PP2 abolished the effect of MPH on response inhibition. (A) Scheme of the experimental procedure. (B)Effects of MPH (1.0 mg/kg) and saline on the SSRTs of the longer-SSRT rats (Slow group) pretreated with PP2, a Src-protein tyrosine kinase (Src-PTKs) inhibitor ( $n=6-8)$. (C) Plot of the normalized SSRT before versus after MPH or saline treatment for each rat pretreated with PP2. ${ }^{\text {ns }} P>0.05$ for PP2+MPH and PP2+saline, permutation test. (D) and (E) Go reaction time (D) and trial accuracies (E) were not significantly changed after the treatment.

receptor function in the PFC.

Intra-PFC inhibition of AMPAR abolishes the effect of MPH on response inhibition

Next, we aimed to determine the contribution of glutamate receptors to the effect of $\mathrm{MPH}$ on response inhibition. Synaptic transmissions mediated by a-amino-3-hydroxy-5-methyl4-isoxazolepropionic acid receptor (AMPAR) and $N$-methyl$D$-aspartic acid receptor (NMDAR) are key to the output of cortical neurons. Considering that the effect of $\mathrm{MPH}$ on response inhibition remained after $3 \mathrm{~h}$ when the enhancement of NMDAR-mediated transmission by MPH did not exist, we speculated that AMPARs within the PFC are implicated in the effect of $\mathrm{MPH}$ on response inhibition. As shown in Figure 5 , the intra-PFC infusion of NASPM $(0.3 \mathrm{mmol} / \mathrm{L}, 0.5 \mu \mathrm{L}$ per side), a selective $\mathrm{Ca}^{2+}$-permeable AMPAR antagonist, had no effect on the SSRTs of the Slow group $(P>0.05, n=6)$; however, when NASPM was administered after the in vivo infusion of MPH $(1.0 \mathrm{mg} / \mathrm{kg})$, the suppression effect of MPH on the SSRTs disappeared $(P>0.05, n=5)$ (Figure 5B). Normalized SSRTs with or without intra-PFC infusion of NASPM after in vivo $\mathrm{MPH}$ infusion showed an even distribution along the diagonal ( $P>0.05$; Figure 5C). Similarly, the Go RT (Figure 5D) and behavior accuracy (Figure 5E) of the Slow group were not changed by these treatments $(P>0.05)$. NASPM was infused into the prelimbic area of the PFC, as shown in Figure 5F.

These results suggested that the prefrontal AMPARs were involved in the effect of MPH on response inhibition in rats.

\section{Discussion}

Although imaging studies have revealed that MPH enhances the neural activities of the cortex (including the PFC) during response inhibition tasks, the mechanism underlying its actions is unknown. In the present study, we used a rodent nose-poking stop-signal task (SST) to examine the effect of $\mathrm{MPH}$ on response inhibition. The stop signal reaction time (SSRT), which reflects the capacity of response inhibition control, is the covert latency to stop a pre-potent action. We determined that MPH $(0.5-1.0 \mathrm{mg} / \mathrm{kg})$ specifically improved response inhibition in rats with longer SSRTs (Slow group) but not with shorter SSRTs (Fast group). We also determined that the effects of MPH on the response inhibition of the Slow group were abolished by the pre-inhibition of glutamate receptor trafficking or intra-PFC inhibition of AMPA receptors. These results suggested that prefrontal AMPARs are involved in the effect of MPH on response inhibition in rats.

Our data showed a baseline-dependent effect of MPH on response inhibition by which the MPH-induced improvement in response inhibition was observed only in rats with longer SSRTs (Slow group). This result was consistent with findings from a previous report ${ }^{[13]}$; however, we did not find a MPHinduced deficit in rats with shorter SSRTs (Fast group). This discrepancy may have been due to the different strains of rats and the different behavior setups used in these two studies. Different strains of rats may have different sensitivities to the changes in dopamine and norepinephrine levels in response to $\mathrm{MPH}$ in the brain $^{[38]}$. In addition, the nose-poking action can 
A
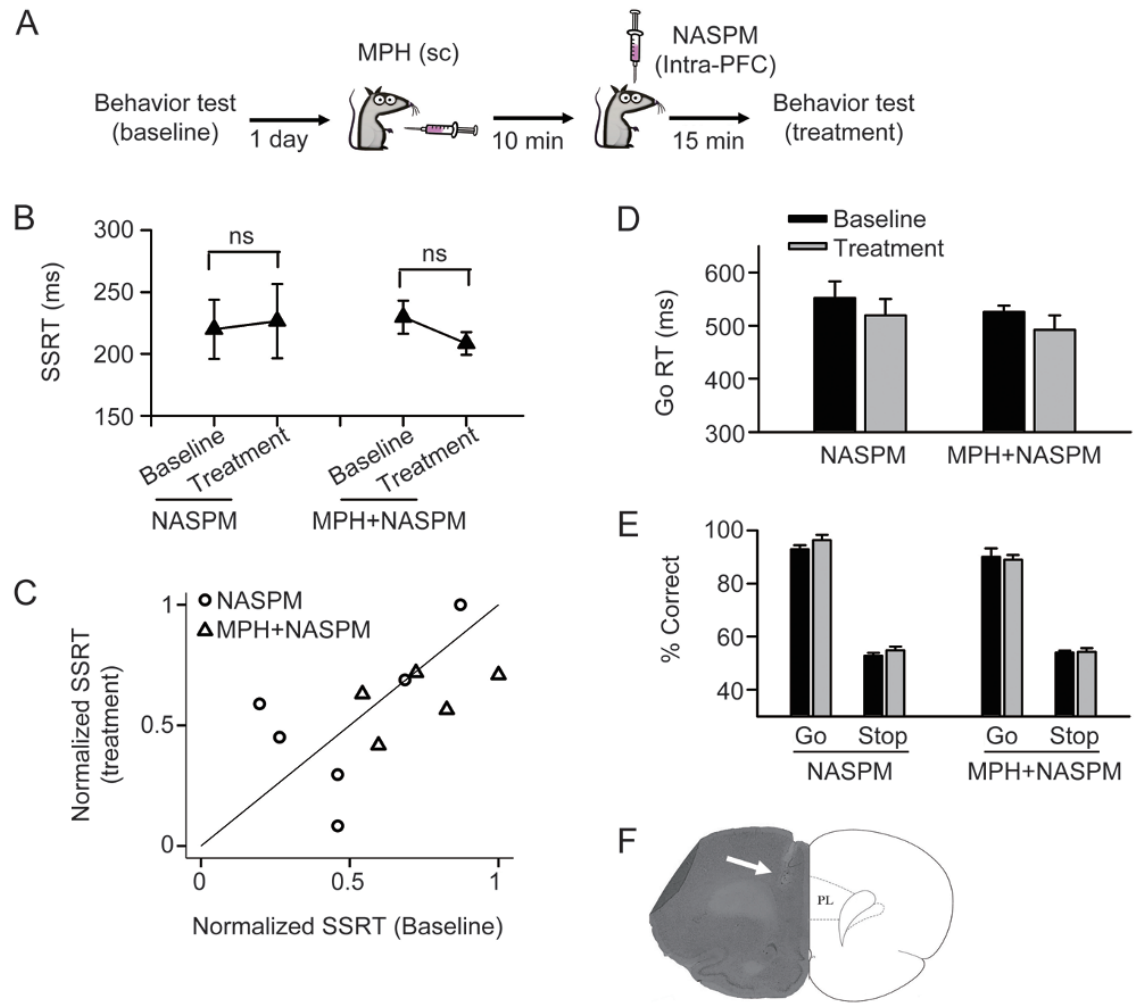

Figure 5. NASPM abolished the effect of MPH on response inhibition. (A) Scheme of the experimental procedure. (B) Intra-PFC infusion of NASPM, a selective $\mathrm{Ca}^{2+}$-permeable AMPAR antagonist, abolished the effect of MPH on the SSRTs of rats with longer SSRTs (Slow group). ( $\left.n=5-6\right)$. (C) Normalized SSRTs in both groups were evenly distributed along the diagonal. ${ }^{n} \mathrm{P}>0.05$ for MPH+NASPM, permutation test. (D, E) Go reaction time (Go RT) (D) and trial accuracies (E) were not significantly changed after the treatments. (F) An example section shows the infusion site of NASPM in the PFC, as indicated by the arrow. PL, prelimbic area.

be performed much faster than lever pressing, thus decreasing the action delay of rats and potentially leading to a more accurate SSRT estimation.

Despite the widespread use of MPH in the treatment of ADHD, approximately $30 \%$ of individuals with ADHD are not responsive to $\mathrm{MPH}^{[39,40]}$. The Fast group, which had shorter SSRTs in our study, may resemble those patients. Several studies suggested that fewer dopamine transporters are expressed in non-responsive patient ${ }^{[41]}$, and individual differences, especially genetic factors, may also partially explain this unexpected outcome ${ }^{[42,43]}$. More work is necessary to identify the different features of these two populations, eg, the amount or function of AMPA receptors in the PFC.

The dose effects of MPH on cognitive function have been well characterized in rats. At the dose of $0.25 \mathrm{mg} / \mathrm{kg}, \mathrm{MPH}$ has been proposed to improve working memory ${ }^{[16]}$; however, our results showed that $0.25 \mathrm{mg} / \mathrm{kg}$ MPH had no effect on response inhibition in rats. Moreover, the $\mathrm{MPH}$-induced improvement in response inhibition still persisted at $3 \mathrm{~h}$ after treatment, whereas the improvement of working memory by $1.0 \mathrm{mg} / \mathrm{kg} \mathrm{MPH} \mathrm{disappeared} \mathrm{(unpublished} \mathrm{data).} \mathrm{These}$ discrepancies suggested that distinct mechanisms underlie the $\mathrm{MPH}$-induced improvements in working memory and response inhibition.

The behavioral effects of the therapeutic dose of MPH have been linked to MPH-potentiated glutamate receptor expression and function in PFC neurons ${ }^{[29]}$. A previous report has shown that response inhibition training increases the contribution of AMPAR at excitatory synapses in the prefrontal cortex $^{[44]}$. In the present study, we used PP2 to prevent the $\mathrm{MPH}$-induced upregulation of glutamate receptor expression. PP2 is a Src-family tyrosine kinase inhibitor used to block glutamate receptor trafficking ${ }^{[36,37,45]}$. Co-infusion of MPH with PP2 diminished the increase in the expression and function of glutamate receptors in PFC neurons (data not shown), and the effect of MPH on response inhibition disappeared in rats that were pre-treated with PP2. AMPARs and NMDARs are major glutamate receptors in the cortex. The present study provides evidence that AMPARs within the PFC mediate the effect of $\mathrm{MPH}$ on response inhibition. We locally administered a subeffective dose of a selective AMPAR antagonist, NASPM, into the PFC of saline-treated rats, and this treatment did not affect response inhibition; however, when NASPM was administered to the MPH-treated rats, the improvement effect of MPH was abolished. These results indicated an important role of AMPA receptors in response inhibition. Consistent with this finding, a recent study has also reported a decrease in the expression and function of AMPAR in the PFC in an ADHD rat model ${ }^{[46]}$. We speculated that the therapeutic effect of MPH on response inhibition enhances the expression of AMPAR 
on the cell surface in the PFC. However, further studies are needed to confirm the underlying mechanism of enhanced AMPAR expression by MPH.

The PFC has extensive interconnections with subcortical regions, especially the basal ganglia structures such as the striatum, global pallidus, and subthalamic nucleus ${ }^{[1,47-49]}$. It is well known that the basal ganglia, as a behavior switch, is indispensable in response inhibition ${ }^{[30,50-52]}$. The potentiation of AMPAR function in PFC neurons by MPH may enhance the top-down control of the PFC over the basal ganglia, thereby promoting response inhibition ${ }^{[53,54]}$.

No significant effects of MPH on the Go RT or behavior accuracies were observed, thus suggesting that the doses of MPH used in the present study did not interfere with the correct execution of the stop-signal task. The Go RT represents the speed of response and requires the integrity of movement control. Our data suggested that MPH did not affect head movements, and the motivations of the rats performing the task were intact. Behavior accuracies remained unaffected, thus indicating that the task rules were also actively maintained.

In summary, this study demonstrated that AMPARs in the PFC are involved in the effect of MPH on response inhibition. This advancement in understanding of the complex actions of MPH may aid in the development of potential treatments for impulsivity-related diseases.

\section{Acknowledgements}

This work was supported by grants from the National Natural Science Foundation of China (№ 31571098) to Xue-han. ZHANG.

\section{Author contribution}

Xue-han ZHANG and Yu-qiu ZHANG designed research; Dong-dong ZHANG performed research and analyzed data; Dong-dong ZHANG and Xue-han ZHANG wrote the paper.

\section{References}

1 Stuphorn V. Neural mechanisms of response inhibition. Curr Opin Behav Sci 2015; 1: 64-71.

2 Hughes ME, Fulham WR, Johnston PJ, Michie PT. Stop-signal response inhibition in schizophrenia: Behavioural, event-related potential and functional neuroimaging data. Biol Psychol 2012; 89: 220-31.

3 Morein-Zamir S, Robbins TW. Fronto-striatal circuits in responseinhibition: Relevance to addiction. Brain Res 2015; 1628: 117-29.

4 Lei H, Zhu X, Fan J, Dong J, Zhou C, Zhang X, et al. Is impaired response inhibition independent of symptom dimensions in obsessivecompulsive disorder? Evidence from ERPs. Sci Rep 2015; 5: 10413.

5 Bari A, Robbins TW. Inhibition and impulsivity: Behavioral and neural basis of response control. Prog Neurobiol 2013; 108: 44-79.

6 Konrad K, GÜNther T, Hanisch C, Herpertz-Dahlmann B. Differential effects of methylphenidate on attentional functions in children with Attention-Deficit/Hyperactivity Disorder. J Am Acad Child Adolescent Psychiatry 2004; 43: 191-8.

7 Biederman J. Attention-deficit/hyperactivity disorder: a selective overview. Biol Psychiatry 2005; 57: 1215-20.

8 Boonstra AM, Kooij JJS, Oosterlaan J, Sergeant JA, Buitelaar JK. Does methylphenidate improve inhibition and other cognitive abilities in adults with childhood-onset ADHD? J Clin Exp Neuropsychol 2005; 27: 278-98.

9 Aron AR, Dowson JH, Sahakian BJ, Robbins TW. Methylphenidate improves response inhibition in adults with attention-deficit/ hyperactivity disorder. Biol Psychiatry 2003; 54: 1465-8.

10 DeVito EE, Blackwell AD, Clark L, Kent L, Dezsery AM, Turner DC, et al. Methylphenidate improves response inhibition but not reflectionimpulsivity in children with attention deficit hyperactivity disorder (ADHD). Psychopharmacology 2008; 202: 531-9.

11 Overtoom CCE, Bekker EM, van der Molen MW, Verbaten MN, Kooij JJS, Buitelaar JK, et al. Methylphenidate restores link between stop-signal sensory impact and successful stopping in adults with attention-deficit/hyperactivity disorder. Biol Psychiatry 2009; 65: 614-9.

12 Smith ME, Farah MJ. Are prescription stimulants "smart pills"? The epidemiology and cognitive neuroscience of prescription stimulant use by normal healthy individuals. Psychol Bull 2011; 137: 717-41.

13 Eagle DM, Tufft MRA, Goodchild HL, Robbins TW. Differential effects of modafinil and methylphenidate on stop-signal reaction time task performance in the rat, and interactions with the dopamine receptor antagonist cis-flupenthixol. Psychopharmacology 2007; 192: 193206.

14 Iversen L. Neurotransmitter transporters and their impact on the development of psychopharmacology. Br J Pharmacol 2006; 147: S82-S88.

15 Volkow ND, Wang G-J, Tomasi D, Kollins SH, Wigal TL, Newcorn JH, et al. Methylphenidate-elicited dopamine increases in ventral striatum are associated with long-term symptom improvement in adults with attention deficit hyperactivity disorder. J Neurosci 2012; 32: 841-9.

16 Berridge CW, Devilbiss DM, Andrzejewski ME, Arnsten AFT, Kelley $\mathrm{AE}$, Schmeichel B, et al. Methylphenidate preferentially increases catecholamine neurotransmission within the prefrontal cortex at low doses that enhance cognitive function. Biol Psychiatry 2006; 60: 1111-20.

17 Miller EK, Cohen JD. An integrative theory of prefrontal cortex function. Annu Rev Neurosci 2001; 24: 167-202.

18 Kim H, Ährlund-Richter S, Wang X, Deisseroth K, Carlén M. Prefrontal parvalbumin neurons in control of attention. Cell 2016; 164: 208-18.

19 Narayanan NS, Laubach M. Inhibitory control: mapping medial frontal cortex. Curr Biol 2017; 27: R148-R50.

20 Guo F, Zhang Q, Zhang B, Fu Z, Wu B, Huang C, et al. Burst-firing patterns in the prefrontal cortex underlying the neuronal mechanisms of depression probed by antidepressants. Eur J Neurosci 2014; 40: 3538-47.

21 Bari A, Mar AC, Theobald DE, Elands SA, Oganya KCNA, Eagle DM, et al. Prefrontal and monoaminergic contributions to stop-signal task performance in rats. J Neurosci 2011; 31: 9254-63.

22 Dambacher F, Sack AT, Lobbestael J, Arntz A, Brugman S, Schuhmann T. The role of right prefrontal and medial cortex in response inhibition: interfering with action restraint and action cancellation using transcranial magnetic brain stimulation. J Cogn Neurosci 2014; 26: 1775-84.

23 Hardung S, Epple R, Jäckel Z, Eriksson D, Uran C, Senn V, et al. A functional gradient in the rodent prefrontal cortex supports behavioral inhibition. Curr Biol 2017; 27: 549-55.

24 Krämer UM, Solbakk A-K, Funderud I, Løvstad M, Endestad T, Knight RT. The role of the lateral prefrontal cortex in inhibitory motor control. Cortex 2013; 49: 837-49.

25 Munakata Y, Herd SA, Chatham CH, Depue BE, Banich MT, O’Reilly RC. A unified framework for inhibitory control. Trends Cognitive Sci 2011; 15: 453-9. 
26 Matuskey D, Luo X, Zhang S, Morgan PT, Abdelghany O, Malison $\mathrm{RT}$, et al. Methylphenidate remediates error-preceding activation of the default mode brain regions in cocaine-addicted individuals. Psychiatry Res 2013; 214: 116-21.

27 Pauls AM, O'Daly OG, Rubia K, Riedel WJ, Williams SCR, Mehta MA. Methylphenidate effects on prefrontal functioning during attentionalcapture and response inhibition. Biol Psychiatry 2012; 72: 142-9.

28 Moeller SJ, Honorio J, Tomasi D, Parvaz MA, Woicik PA, Volkow ND, et al. Methylphenidate enhances executive function and optimizes prefrontal function in both health and cocaine addiction. Cereb Cortex 2014; 24: 643-53.

29 Cheng J, Xiong Z, Duffney ப, Wei J, Liu A, Liu S, et al. Methylphenidate exerts dose-dependent effects on glutamate receptors and behaviors. Biol Psychiatr 2014; 76: 953-62.

30 Hikosaka 0 , Isoda M. Switching from automatic to controlled behavior: cortico-basal ganglia mechanisms. Trends Cognitive Sci 2010; 14: 154-61.

31 Thakkar KN, Heiligenberg FMZvd, Kahn RS, Neggers SFW. Frontalsubcortical circuits involved in reactive control and monitoring of gaze. J Neurosci 2014; 34: 8918-29.

32 Aron AR, Herz DM, Brown P, Forstmann BU, Zaghloul K. Frontosubthalamic circuits for control of action and cognition. J Neurosci 2016; 36: 11489-95.

33 Wessel JR, Aron AR. On the globality of motor suppression: unexpected events and their influence on behavior and cognition. Neuron 2017; 93: 259-80.

34 Zhang Q, Guo F, Fu ZW, Zhang B, Huang CG, Li Y. Timosaponin derivative $Y Y-23$ acts as a non-competitive NMDA receptor antagonist and exerts a rapid antidepressant-like effect in mice. Acta Pharmacol Sin 2016; 37: 166-76.

35 Logan GD, Cowan WB. On the ability to inhibit thought and action: A theory of an act of control. Psychol Rev 1984; 91: 295-327.

36 Bonifacino JS, Traub LM. Signals for sorting of transmembrane proteins to endosomes and lysosomes. Annu Rev Biochem 2003; 72 : 395-447.

37 Groveman BR, Xue S, Marin V, Xu J, Ali MK, Bienkiewicz EA, et al. Roles of the $\mathrm{SH} 2$ and $\mathrm{SH} 3$ domains in the regulation of neuronal Src kinase functions. FEBS J 2011; 278: 643-53.

38 Yoon YW, Lee DH, Lee BH, Chung K, Chung JM. Different strains and substrains of rats show different levels of neuropathic pain behaviors. Exp Brain Res 1999; 129: 167-71.

39 Cantwell DP. Attention deficit disorder: a review of the past 10 years. J Am Acad Child Adolesc Psychiatry 1996; 35: 978-87.

40 Krause J, Fougere $\mathrm{Cl}$, Krause KH, Ackenheil M, Dresel SH. Influence of striatal dopamine transporter availability on the response to methylphenidate in adult patients with ADHD. Eur Arch Psychiatry Clin
Neurosci 2005; 255: 428-31.

41 Winsberg BG, Comings DE. Association of the dopamine transporter gene (DAT1) with poor methylphenidate response. J Am Acad Child Adolesc Psychiatry 1999; 38: 1474-7.

42 McGough JJ, McCracken JT, Loo SK, Manganiello M, Leung MC, Tietjens JR, et al. A candidate gene analysis of methylphenidate response in attention-deficit/hyperactivity disorder. J Am Acad Child Adolesc Psychiatry 2009; 48: 1155-64.

43 Pagerols M, Richarte V, Sánchez-Mora C, Garcia-Martínez I, Corrales $M$, Corominas $M$, et al. Pharmacogenetics of methylphenidate response and tolerability in attention-deficit/hyperactivity disorder. Pharmacogenomics J 2017; 17: 98-104.

44 Hayton SJ, Lovett-Barron M, Dumont EC, Olmstead MC. Target-specific encoding of response inhibition: increased contribution of AMPA to NMDA receptors at excitatory synapses in the prefrontal cortex. J Neurosci 2010; 30: 11493-500.

45 Kalia LV, Salter MW. Interactions between Src family protein tyrosine kinases and PSD-95. Neuropharmacology 2003; 45: 720-8.

46 Cheng J, Liu A, Shi MY, Yan Z. Disrupted glutamatergic transmission in prefrontal cortex contributes to behavioral abnormality in an animal model of ADHD. Neuropsychopharmacology 2017; 42: 2096-104.

47 Duan CA, Erlich JC, Brody CD. Requirement of prefrontal and midbrain regions for rapid executive control of behavior in the rat. Neuron 2015; 86: 1491-503.

48 Burguière $E$, Monteiro $P$, Feng G, Graybiel AM. Optogenetic stimulation of lateral orbitofronto-striatal pathway suppresses compulsive behaviors. Science 2013; 340: 1243-6.

49 Morris LS, Baek K, Voon V. Distinct cortico-striatal connections with subthalamic nucleus underlie facets of compulsivity. Cortex 2017; 88: $143-50$.

50 Kreitzer AC, Malenka RC. Striatal plasticity and basal ganglia circuit function. Neuron 2008; 60: 543-54.

51 Maurice N, Deniau JM, Glowinski J, Thierry AM. Relationships between the prefrontal cortex and the basal ganglia in the rat: physiology of the corticosubthalamic circuits. J Neurosci 1998; 18 : 9539-46.

52 Aron AR, Durston S, Eagle DM, Logan GD, Stinear CM, Stuphorn V. Converging evidence for a fronto-basal-ganglia network for inhibitory control of action and cognition. J Neurosci 2007; 27: 11860-4.

53 Narayanan NS, Laubach M. Top-Down control of motor cortex ensembles by dorsomedial prefrontal cortex. Neuron 2006; 52: $921-$ 31.

54 Rae CL, Hughes LE, Anderson MC, Rowe JB. The prefrontal cortex achieves inhibitory control by facilitating subcortical motor pathway connectivity. J Neurosci 2015; 35: 786-94. 